\title{
Distrofia miotónica congénita en una Unidad de Cuidados Intensivos Neonatales: serie de casos
}

\author{
Congenital myotonic dystrophy in a Neonatal Intensive Care Unit: case series
}

\author{
Dra. Sara Domingues ${ }^{a}$, Dra. Clara Alves Pereira ${ }^{a}$,Dra. Ángela Machado ${ }^{a}$,Dra. Sandra Pereira ${ }^{a}$, \\ Dra. Leonilde Machado ${ }^{b}$ Dra. Carla Fragac ${ }^{c}$ Dr. Abílio Oliveira ${ }^{a}$ Dra. Isabel Vale ${ }^{a}$, Dr. Ilídio Quelhas ${ }^{a}$
}

\section{RESUMEN}

La distrofia miotónica de Steinert es una enfermedad multisistémica, autosómica dominante, con un amplio espectro de gravedad y manifestaciones clínicas. La forma más grave es aquella que se manifiesta en el periodo neonatal, llamada distrofia miotónica congénita. Se destaca la hipotonía global al nacer y el compromiso de la función respiratoria. Las complicaciones son frecuentes, principalmente, retraso del desarrollo psicomotor, del crecimiento pondoestatural, dificultades alimentarias y constipación. Se asocia a un mal pronóstico, con una mortalidad global de hasta un $50 \%$ de los niños gravemente afectados.

Presentamos cinco casos de distrofia miotónica congénita con el objetivo de describir manifestaciones clínicas, métodos diagnósticos, tratamiento y pronóstico. Los datos existentes en la literatura sobre el desarrollo psicomotor, complicaciones y pronóstico de los supervivientes con distrofia miotónica congénita son pocos. En nuestra serie de casos, las limitaciones psicomotoras presentadas son significativas.

Palabras clave: distrofia miotónica tipo 1, hipotonía muscular, insuficiencia respiratoria, mortalidad, retraso del desarrollo psicomotor.

\section{SUMMARY}

Steinert myotonic dystrophy is a multisystemic disease, autosomal dominant, with a wide spectrum of severity and clinical manifestations. The most severe form is one that manifests in the neonatal period, called congenital myotonic dystrophy. This condition is distinguished by overall hypotonia at birth and respiratory function compromise. Complications are frequent, mainly psychomotor development delay, growth failure, food difficulties and constipation. It is associated with a poor prognosis, with an overall mortality of up to $50 \%$ of severely affected children.

We present five patients with congenital myotonic dystrophy in order to describe clinical manifestations, diagnosis, treatment and prognosis. Existing data in the literature on psychomotor development, complications and prognosis of survivors with

a. Unidad de Cuidados Intensivos Neonatales.

b. Servicio de Pediatría.

c. Servicio de Neurología.

Centro Hospitalar do Tâmega e Sousa, E. P. E.,

Unidade Padre Américo. Penafiel, Portugal.

Correspondencia:

Sara Domínguez: e-mail: saradomingues@hotmail.com

Conflicto de intereses: Ninguno que declarar.

Recibido: 13-6-2013

Aceptado: 29-10-2013 congenital myotonic dystrophy are scarce. In our case studies, we have found significant chronic psychomotor limitations. Key words: myotonic dystrophy type 1, mortality, muscular hypotonia, psychomotor development delay, respiratory insufficiency.

http:/ /dx.doi.org/10.5546/aap.2014.e18

\section{INTRODUCCIÓN}

La distrofia miotónica fue descrita por primera vez en 1909 por Steinert. ${ }^{1}$ Es la segunda distrofia muscular más común entre la población europea ${ }^{2}$ y su prevalencia ronda los 1:20 000 casos en la población en general ${ }^{1,3-5}$

La distrofia miotónica tipo 1 (DM1) es una enfermedad multisistémica, autosómica dominante, con expresión variable..$^{3,4,6-8}$ Resulta de la expansión del número de repeticiones del trinucleótido citosina, timina, guanina (CTG) (más de 50) en el gen de la proteína quinasa de distrofia miotónica (DMPK, por sus siglas en inglés) del cromosoma 19.2,4 La expansión provoca inestabilidad alélica miótica y meiótica, ${ }^{3,4}$ lo que permite el aumento del número de repeticiones a lo largo de generaciones (fenómeno de anticipación), ${ }^{3,5}$ que se asocia a una mayor precocidad y gravedad de la enfermedad en las formas neonatales. ${ }^{3}$ Una segunda forma (DM2) se asocia con la expansión inestable de CTG en el gen de la proteína Zinc finger 9 del cromosoma 3q21; su fisiopatología y patogenia son similares a las de la DM1, pero tiene un fenotipo más benigno, no relacionado con formas congénitas. ${ }^{2,3,9,10}$

La distrofia miotónica congénita (DMC) es la forma más grave de DM1 y tiene una incidencia estimada a nivel mundial de 3-15:100 000 nacidos vivos. ${ }^{11}$ La transmisión es, en más del $90 \%$ de los casos, de origen materno ${ }^{2,12}$ por probable inestabilidad meiótica aumentada durante la ovogénesis, en relación con la espermatogénesis. ${ }^{10}$

A continuación, describimos cinco casos de DMC.

\section{Casos clínicos}

Presentamos cinco pacientes con DMC internados en la Unidad de Cuidados Intensivos 
Neonatales del Centro Hospitalar Tâmega e Sousa entre enero de 2008 y diciembre de 2011. Todos los recién nacidos $(\mathrm{RN})$ presentaron hipotonía global e insuficiencia respiratoria: cuatro, con ausencia de movimientos respiratorios espontáneos al nacer, por lo que se inició la asistencia respiratoria mecánica (ARM); el otro caso presentaba movimientos respiratorios débiles al nacer y permaneció bajo asistencia respiratoria no invasiva. Se verificaron dos fallecimientos y los restantes casos presentan retraso del desarrollo psicomotor. La descripción de los pacientes se resume en la Tabla 1.

\section{DISCUSIÓN}

Habitualmente, la DM1 se manifiesta a partir de la segunda década de vida ${ }^{8}$ con debilidad muscular distal lentamente progresiva, ${ }^{2}$ que dificulta la realización de tareas que requieren una buena destreza de las manos, y la caída del pie. ${ }^{3,8,11}$ La facies es característica, con atrofia de los músculos faciales y ptosis. ${ }^{3}$ La miotonía (retraso en la relajación muscular después de la contracción) es el signo cardinal y se puede demostrar a través de un apretón o contracción sostenidos de manos. ${ }^{2,3}$

En nuestra serie, todas las madres presentaban facies sugestiva, miotonía y retraso cognitivo, pero solo en un caso existía conocimiento familiar de la enfermedad.

Las principales causas de muerte son de etiología respiratoria (insuficiencia respiratoria neuromuscular progresiva y neumonía) o cardíaca (muerte súbita debido a perturbaciones graves de la conducción o taquiarritmias y la enfermedad cardiovascular)..$^{8-11}$

En la DMC, la hipotonía se puede manifestar antes del nacimiento, con referencia frecuente a la reducción de los movimientos fetales, presentación fetal distócica e polihidramnios (por trastornos deglutorios). ${ }^{3,7-9,12} \mathrm{El}$ parto normalmente es prolongado, con necesidad de intervención instrumental. ${ }^{13,14} \mathrm{Al}$ nacer, los individuos afectados presentan ya una facies sugestiva, poco expresiva, alargada por atrofia de los músculos maseteros y temporales, con el labio superior en $\mathrm{V}$ invertida y paladar ojival. ${ }^{13}$ También pueden presentar otras señales secundarias a la inmovilidad e hipotonía, como artrogriposis y criptorquidia. ${ }^{7,12}$ Se destaca la hipotonía global y el compromiso de la función respiratoria con necesidad de asistencia respiratoria mecánica (ARM) en aproximadamente el $80 \%$ de los casos. ${ }^{8}$ La necesidad de soporte ventilatorio inferior a 30 días está asociada a menor mortalidad y morbilidad. ${ }^{2,10}$ Las complicaciones son frecuentes: principalmente, retraso del desarrollo psicomotor, del crecimiento pondoestatural, dificultades alimentarias y constipación. ${ }^{3,9}$ Varios autores describen una mejora progresiva del tono y de la fuerza muscular; ${ }^{7}$ algunos niños adquieren la capacidad de marcha autónoma. ${ }^{3,5,9,15} \mathrm{La}$ mortalidad es alta y puede alcanzar al $50 \%$ de los niños gravemente afectados, ${ }^{7,8}$ por lo general, por insuficiencia respiratoria ${ }^{10}$ (debido a la debilidad del diafragma y anormalidad cerebral de control de la respiración). ${ }^{2,3,8}$

En nuestra serie, todos los $\mathrm{RN}$ requieren soporte ventilatorio al nacer; este porcentaje es superior al descrito. ${ }^{8}$ Solo en un caso, la necesidad de soporte ventilatorio fue inferior a 30 días; ese niño tuvo un desarrollo psicomotor adecuado a la edad, excepto en la capacidad locomotora. Este hecho corrobora el mejor pronóstico de los niños con necesidad de soporte ventilatorio por periodos inferiores. ${ }^{2,10}$ Los restantes casos presentan retraso de desarrollo grave. El retraso cognitivo puede deberse a la acumulación de alelo DMPK mutante en las neuronas corticales, con efecto tóxico directo. ${ }^{2,3,9,15} \mathrm{C}$. Turner et ál. ${ }^{11}$ mostraron la implicación cognitiva en la DM1, de acuerdo con nuestros resultados. Como era de esperar, la insuficiencia respiratoria fue la principal causa de muerte en estos niños. ${ }^{10}$

Anomalías de la conducción cardíaca, cataratas, hipogammaglobulinemia y resistencia a la insulina se producen comúnmente en estos pacientes, por lo que debe realizarse al menos un electrocardiograma cada año, así como la evaluación periódica oftalmológica, los estudios de inmunoglobulinas séricas y la prueba de tolerancia a la glucosa. ${ }^{2,8,9}$

El metilxantine puede utilizarse para tratar la miotonía, ${ }^{9-11,15}$ pero este no se consideró en nuestra serie.

El diagnóstico de DMC siempre se debe considerar en la evaluación del RN con hipotonía, incluso cuando la historia familiar de enfermedad es desconocida. Es esencial un buen interrogatorio familiar en busca de cataratas, calvicie, azoospermia, alteraciones cardíacas, retraso cognitivo, trastornos musculares y muertes en el periodo neonatal. ${ }^{7-9}$ Frente a esta hipótesis diagnóstica, el examen físico de la madre puede ser revelador (presencia de fenómeno miotónico). ${ }^{7,8}$

Es la determinación de la expansión del triplete CTG lo que confirma el diagnóstico de DMC. ${ }^{12}$ Los niveles de creatincinasa en suero 
TABLa 1. Resumen descriptivo de los casos

\begin{tabular}{|c|c|c|c|c|c|}
\hline \multirow[b]{2}{*}{ Variables } & \multicolumn{5}{|c|}{ Caso } \\
\hline & 1 & 2 & $3^{\mathrm{a}}$ & $4^{\mathrm{a}}$ & 5 \\
\hline Sexo & M & $\mathrm{F}$ & M & $\mathrm{F}$ & $\mathrm{F}$ \\
\hline Edad gestacional (semanas) & 32 & 32 & 37 & 37 & 33 \\
\hline Parto (tipo/presentación) & Ces./C & Ces./P & Ces./C & Ces. $/ C^{b}$ & Ces./P \\
\hline Peso al nacer (gramos) & 1180 & 1420 & 2100 & 2630 & 1740 \\
\hline Pequeño para la edad gestacional & + & + & + & - & + \\
\hline $\operatorname{Apgar}\left(1^{\prime} / 5^{\prime} / 10^{\prime}\right)$ & $4 / 6 / 6$ & $1 / 4 / i ?$ & $4 / 8 / 8$ & $2 / 5 / 6$ & $4 / 7 / 7$ \\
\hline \multicolumn{6}{|l|}{ Manifestaciones clínicas } \\
\hline Hidramnios & - & + & + & + & + \\
\hline Movimientos fetales disminuidos & + & + & + & + & - \\
\hline Hipotonía global & + & + & + & + & + \\
\hline Facies sugestiva & + & + & + & + & - \\
\hline Paladar ojival & - & + & + & - & - \\
\hline Criptorquidia & + & - & + & - & - \\
\hline Deformidad de los pies & + & - & + & - & - \\
\hline \multicolumn{6}{|l|}{ Estudio genético } \\
\hline Otros familiares afectados & Madre/O & Madre/H/O & Madre/H/O & Madre/H/O & Madre/O \\
\hline Diagnóstico & Molecular & Molecular (prenatal) & Molecular & Presuntivo & Molecular \\
\hline Número de repeticiones CTG madre/RN & $1300 / 1800$ & --- & $350-600 / 1200$ & $350-600 / i ?$ & -- \\
\hline \multicolumn{6}{|l|}{ Evolución } \\
\hline Soporte ventilatorio (días) & 365 & 62 & 11 & 9 & 38 \\
\hline Alimentación oral total (día) & 120 & 41 & 21 & --- & --- \\
\hline Complicaciones & Oc., R, GI, Ort., DPM & DPM & Oc., GI, Ort., DPM & --- & --- \\
\hline Muerte (día) & --- & --- & -- & 9 & 38 \\
\hline Edad cronológica/desarrollo (meses) ${ }^{c}$ & $45 /$ & & & & \\
\hline $10-12^{\mathrm{d}}$ & $21 /$ & & & & \\
\hline $8-10-12^{\mathrm{e}}$ & $17 /$ & & & & \\
\hline $10-15-18^{f}$ & --- & --- & & & \\
\hline
\end{tabular}

Abreviaturas y siglas: Ces., cesárea; C., cefálica; DPM, desarrollo psicomotor; F, femenino; M, masculino; H, hermanos;

O, otro familiar; GI, gastrointestinal; Oc., ocular; Ort., ortopédica; P, pélvica; R, respiratoria; RN, recién nacido.

${ }^{a}$ Los pacientes tres y cuatro eran frutos de gestación bigemelar y primos terceros (nietos de un hermano del abuelo materno) del paciente dos.

${ }^{\mathrm{b}}$ El parto fue prolongado; se encontraba en presentación cefálica inicial. Se efectuó reversión interna y posteriormente se extrajo en pelvis. La tomografía computarizada reveló hemorragia intraventricular, supra e infratentorial, así como hemorragia subaracnoidea frontoparietal en probable contexto de hipoxia neonatal.

c Perfil de evaluación del desarrollo de los supervivientes efectuado con recurso a la escala adaptada de "Schedule of Growing Skills II".

${ }^{\mathrm{d}}$ Locomoción: < 10 meses (ausente); control postural activo, manipulación, visión, habla y lenguaje: 10 meses; audición y lenguaje, interacción social, autonomía: 12 meses.

e Control postural activo: 8 meses; locomoción, autonomía: 10 meses; manipulación, audición y lenguaje, habla y lenguaje, interacción social: 12 meses.

f Locomoción: 10 meses; visión, habla y lenguaje, interacción social, autonomía: 15 meses; manipulación, audición y lenguaje: 18 meses. 
pueden ser normales ${ }^{2}$ y no nos va a ser útil practicar al RN un electromiograma, ya que el fenómeno miotónico solo aparece después de los dos años. ${ }^{11}$ La biopsia muscular normalmente no es necesaria para el diagnóstico. ${ }^{2}$

El diagnóstico de DM1 fue confirmado por estudio molecular en cuatro de los casos. En dos de ellos, el conocimiento del número de repeticiones de CTG en la madre $(\sim 1300 \mathrm{y} \sim 350$ $600)$, y RN ( 1800 y 1200, respectivamente) corrobora la asociación entre el aumento del número de repeticiones a lo largo de las generaciones y la mayor precocidad y gravedad de la enfermedad. ${ }^{3}$ Hay que destacar, sin embargo, que el número de repeticiones por sí solo no predice la gravedad individual de la forma de presentación, tal como la madre del caso uno, que presenta más de 1000 repeticiones en la forma adulta de la enfermedad. ${ }^{7}$
En el caso número cuatro, el diagnóstico de DMC fue presuntivo y se basó en el cuadro clínico y la confirmación de la enfermedad en la madre y en el hermano. No obstante, la asfixia perinatal sufrida por este RN puede suscitar dudas diagnósticas. ${ }^{8,13,14}$ Goméz Manchón et ál. ${ }^{14}$ describen un caso semblante a esto, con una forma de presentación grave en que los signos de encefalopatía hipóxico-isquémica enmascaran una probable DMC.

El diagnóstico diferencial en el periodo neonatal incluye aún la miopatía estructural miotubular y el síndrome de Moebius. ${ }^{13}$

Durante un periodo de cuatro años, observamos cinco pacientes con el diagnóstico de DMC, lo que corresponde a una incidencia de aproximadamente 25:100 000 nacidos vivos, superior a la descrita. ${ }^{11}$ La relación de parentesco constatada entre tres de los RN podrá justificar este hallazgo.

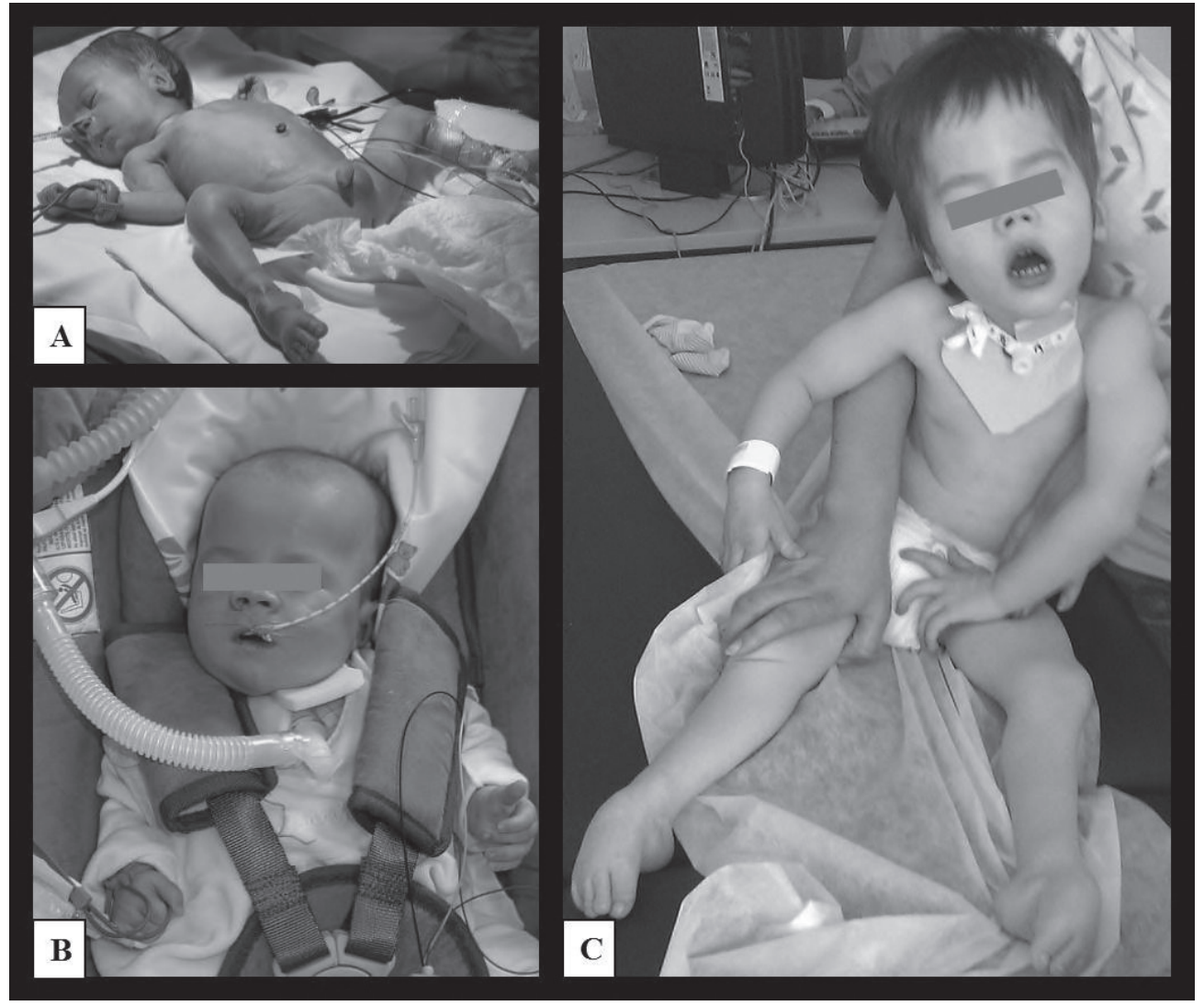

A) Periodo neonatal: se observa hipotonía global, artogriposis de las rodillas, tobillos y puños, y pies equinos.

B) Tres meses: necesidad de ventilación mecánica por cánula de traqueotomía. C) Tres años y nueve meses: se destaca facies miopática característica, con labio superior en V invertida e hipotonía global. 
Figura 2. Caso 2: madre e hija con distrofia miotónica tipo 1

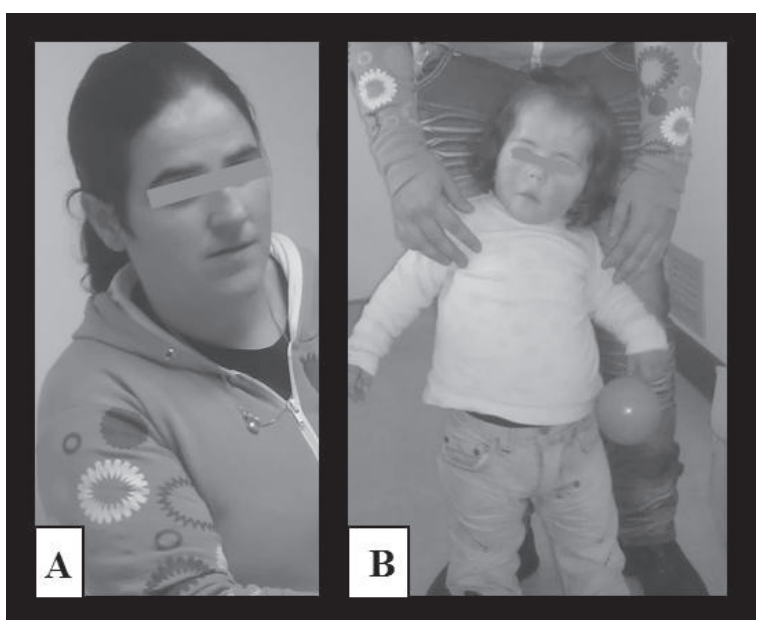

A) Forma de presentación clásica (adulto). B) Hija de 21 meses con DMC; se destaca facies miopática característica, inexpresiva, con labio superior en V invertida e hipotonía; ausencia de equilibrio en la posición supina sin apoyo.
FIGURA 3. Caso 3: madre e hijo con distrofia miotónica tipo 1 (fenómeno de anticipación)

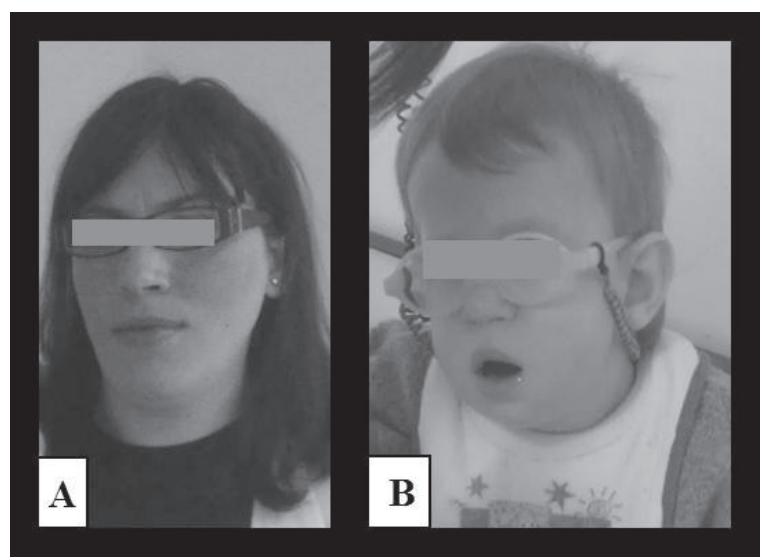

A) Paciente de 33 años, forma de presentación clásica (adulto) ( 350-600 repeticiones de CTG en el gen DMPK).

B) Hijo de 17 meses con distrofia miotónica congénita ( 1200 repeticiones de CTG).
El asesoramiento genético es esencial para la investigación familiar y la orientación adecuada de los pacientes antes de considerar el embarazo (estudio prenatal y preimplantacional). ${ }^{9,11}$

No obstante la evolución tecnológica y técnica, la mortalidad precoz asociada a la DMC permanece alta. ${ }^{10}$ Los datos existentes en la literatura sobre el desarrollo psicomotor, las complicaciones y el pronóstico de los supervivientes con DMC son pocos. En nuestra serie de casos, las limitaciones motoras y cognitivas son significativas.

\section{BIBLIOGRAFÍA}

1. Aviña Fierro JA, Hernández Aviña DA. Síndrome de Steinert neonatal: distrofia miotónica tipo 1 congénita. Arch Pediatr Urug 2009;80(1):33-6.

2. Sarnat HB. Myotonic muscular Dystrophy. En: Kliegman RM, Behrman RE, Jenson HB, Stanton BF, eds. Nelson Textbook of Pediatrics. $18^{a}$ ed.Philadelphia:Saunders Elsevier; 2007.Págs.2544-6.

3. Turner C, Hilton-Jones D. The myotonic dystrophies: diagnosis and management. J Neurol Neurosurg Psychiatry 2010;81(4):358-67.

4. Morales-Montero F, Cuenca-Berger P, Brian-Gago R, Sittenfeld M, et al. Estudio intergeneracional de la mutación que causa la distrofia miotónica de tipo 1 en Costa Rica. Rev Neurol 2003;36:20-25.

5. Bouhour F, Bost M, Vial C. Maladie de Steinert. Presse Med 2007;36:965-71.
6. Bird TD. Myotonic dystrophy type 1. Gene Reviews 2011. [Consulta: 15 de septiembre de 2011]. Disponible en: www. geneclinics.org.

7. Rittler M, Feld V, Montagno M. Distrofia miotónica congénita. Rev Hosp Mat Inf Ramón Sardá 1997;1:34-40.

8. Darras BT, Chad DA. Myotonic dystrophy: etiology, clinical features, and diagnosis. Upto Date 2011. [Consulta: 2 de octubre de 2011]. Disponible en: www.uptodate.com.

9. Johnson NE, Heatwole CR. Myotonic Dystrophy: From Brench to Bedside. Semin Neurol 2012;32:246-254.

10. Darras BT, Chad DA. Myotonic dystrophy: prognosis and management. UptoDate 2011. [Consulta: 2 de octubre de 2011]. Disponible en: www.uptodate.com.

11. Turner C, Hilton-Jones D, Lochmuller H, Hanna MG. MRC Centre for Neuromuscular Diseases 1st(1stDecember 2010), and 2nd (2nd May 2012) myotonic dystrophy workshops, London, UK and the myotonic dystrophy standards of care and national registry meeting, Newcastle, UK July 2011, Neuromuscul Disord 2013. Disponible en: http://dx.doi. org/10.1016/j.nmd.2013.07.006.

12. Lorenzo G. Neurología: trastornos neuromusculares aspectos clínicos. An Pediatr Contin 2007;5(5):245-52.

13. Peredo DE, Hannibal MC. The floppy infant: evaluation of hypotonia. Pediatr Rev 2009;30:66-77.

14. Goméz Manchón M, Beceiro Mosquera J, Olivas López de Soria C, Ripalda MJ, et al. Distrofia miotónica congénita y encefalopatía hipóxico-isquémica. An Pediatr (Barc) 2005;62(1):76-84.

15. Udd B, Krahe R. The myotonic dystrophies: molecular, clinical, and therapeutic challenges. Lancet Neurol 2012;11:891-905. 